\title{
Optimal Waveform Selection for Robust Target Tracking
}

\author{
Fengming Xin, Jinkuan Wang, Qiang Zhao, and Yuhuan Wang \\ School of Information Science and Engineering, Northeastern University, Shenyang 110004, China \\ Correspondence should be addressed to Jinkuan Wang; wjk@mail.neuq.edu.cn
}

Received 15 May 2013; Accepted 28 June 2013

Academic Editor: Bin Wang

Copyright (C) 2013 Fengming Xin et al. This is an open access article distributed under the Creative Commons Attribution License, which permits unrestricted use, distribution, and reproduction in any medium, provided the original work is properly cited.

\begin{abstract}
This paper proposes a new optimal waveform selection algorithm for intelligent target tracking. In radar systems, optimal waveform is inspired by the improvements in performance. When the target is intelligent and tries to escape from detection, it will maximize the estimation error to degrade the target tracking performance. So the conventional tracking algorithms are not suitable for this situation. In this paper, we assume a one-dimension target model which will try to escape the radar detection to degrade the tracking performance. A new optimal waveform selection algorithm is proposed based on game theory for robust tracking. The robust received filter is first reviewed according to zero-sum game with the derivation of estimated state error covariance. The parameters for transmitted waveform that need to be optimized are found to be related to the robust filter. The optimal parameters for transmitted waveform are finally found by the minimization of the trace of the estimated state error covariance. Simulation results show the effectiveness of this new proposed algorithm for optimal waveform selection for intelligent target tracking.
\end{abstract}

\section{Introduction}

Since traditional radar/sonar systems lack adaptivity to the different targets, interference, and clutter without utilizing prior measurements or knowledge, they could not adaptively adjust transmitted waveforms to the variant environment. So, the modern radar/sonar systems need more intelligent ability in order to improve the radar performance. Cognitive radar is proposed as a new generation radar system by Haykin $[1,2]$, which can adaptively and intelligently interrogate a propagation channel using all available knowledge. The most important conclusion of cognitive radar system is that it must be able to adaptively generate and transmit the optimal waveforms to improve the accuracy of the radar system.

There are two strategies of generating optimal waveforms, that is, selection and design. However, it is not clear which one is better. Many researchers focused on the optimal waveform technology for different tasks, for example, target detection, estimation, and target tracking [3-17]. The general method is to find signal/filter pairs to maximize the signal-to-clutter plus interference ratio (SCIR) for detecting the target. Pillai et al. developed an eigensolution for optimal signal/filter pairs for target detection when the target and clutter can be seen as linear time invariant random processes $[3,4]$.
Then they extended this approach to optimize the waveform for target identification. The waveform optimization for target identification is addressed by relating SCIR to the Mahalanobis distance $[5,6]$. Information theoretic approach is also an important tool for the waveform optimization. Bell proposed to maximize the mutual information (MI) between the received signal and target impulse response to optimize the waveform [7]. In [8], the authors introduced the relative entropy to optimal waveform for target identification based upon the synthesis of a sequence of probing signals to maximize classification performance, which can extract as much information as possible from the observations. Kay derived the optimal NP detector firstly, which shows that the NP detection performance does not immediately lead to an obvious signal design criterion so that a divergence criterion is proposed for signal design, also based on the relative entropy in signal input multiple output radar scenario [9]. Goodman et al. adopted sequential hypothesis testing combination with mutual information and maximizing the signal-to-noise ratio (SNR) that decides when hard decision may be made with adequate confidence to design the waveform [10]. By comparing the performance of two different waveform design techniques based on information theory [7] and eigensolution [5], Romero et al. also found the 
relationship between the MI and maximizing the SNR in the context of waveform design for stochastic target [11]. In [12], the authors extended Goodman's method, which considered the target detection before the recognition procedure.

There are two main approaches of designing the optimal waveform for target tracking, that is, the control theoretic and information theoretic approaches. The first one is treated as a control problem, since the parameters of the transmitted waveform as an input vector, which is selected or designed to affect the next observation and the tracker, update in a feedback loop. In [13], the authors created the cost function that includes the parameters of transmitted waveform for the next step. The second one also made use of the mutual information (MI) between the target and the observations $[14,15]$. In [14], the authors designed the libraries where the waveform was selected through maximizing the mutual information (MI) between the target model and observations. Then they extended this method to interacting multiple model trackers for different dynamic models [15]. In [13], Kershaw and Evans used the control theoretic approach to optimize the waveform for one-dimensional target tracking in a feedback loop system. They derived the Cramer-Rao lower bound (CRLB) for estimating error variance from the curvature of the peak of the ambiguity function (AF). then the measurement noise covariance matrix that is related to the parameters of the transmitted waveform can be evaluated from the CRLB in high SNR condition. The minimization of the tracking mean square error and the validation gate volume are performed to select the next transmitted waveform. Kershaw and Evans extended their work in clutter and imperfect detection situation [16]. This method was also introduced to the wideband environment for multiple targets tracking in clutter condition [17].

In this paper, we adopt the control theoretic approach to find the optimal waveform for one dimension target tracking [13]. In this paper, we assume a one-dimension target model which will try to escape the radar detection to degrade the tracking performance. A new optimal waveform selection algorithm is proposed based on game theory for robust tracking [18]. The robust received filter is first reviewed according to zero-sum game with the derivation of estimated state error covariance. The parameters for transmitted waveform that need to be optimized are found to be related to the robust filter. The optimal parameters for transmitted waveform are finally found by the minimization of the trace of the estimated state error covariance. Simulation results show the effectiveness of this new proposed algorithm for optimal waveform selection for intelligent target tracking.

This paper is organized in the following manner. Section 2 reviews the control approach in [13] and presents the problem for target tracking. Section 3 describes the optimal waveform selection for robust target tracking. Section 4 shows the simulation results. The conclusion is summarized in Section 5.

\section{Problem Formulation}

We begin with a brief overview of the control approach for one-dimension target tracking in [13]. In radar/sonar system, the transmitted signal can be written as

$$
s_{T}(t)=\sqrt{2} \operatorname{Re}\left\{\sqrt{E_{T}} \widetilde{s}(t) e^{j \omega_{c} t}\right\},
$$

where $\widetilde{s}(t)$ is the complex envelope, $\omega_{c}$ is the carrier frequency, and $E_{T}$ is the energy of the transmitted signal. When the target exists, the received waveform envelope is

$$
\bar{r}(t)=\sqrt{E_{R}} e^{j \phi_{\widetilde{S}}}\left(t-\tau_{0}\right) e^{j v_{0} t}+\widetilde{n}(t),
$$

where $E_{R}$ is the energy of the received signal. $\widetilde{n}(t)$ is zeromean complex white Gaussian noise with real spectral density $N_{0} / 2 . \tau_{0}$ and $v_{0}$ denote the target time delay and Doppler shift, respectively.

The ambiguity function corresponding to the received waveform in frequency domain is written as

$$
A(\tau, \nu)=\int_{-\infty}^{\infty} S\left(\omega-\frac{\nu}{2}\right) S^{*}\left(\omega+\frac{\nu}{2}\right) e^{-j \omega \tau} d \omega / 2 \pi .
$$

The receiver parameter vector is $\boldsymbol{\alpha}=[\tau, \nu]^{T}$.

The target state model as discrete time is defined by

$$
\mathbf{x}_{k+1}=\mathbf{F x}_{k}+\mathbf{G w}_{k} .
$$

The measurement vector equation is given as

$$
\mathbf{y}_{k}=\mathbf{H x}_{k}+\mathbf{n}_{k},
$$

where $\mathbf{y}_{k}=[\mathbf{r}, \dot{\mathbf{r}}]$ denotes the measurement vector. $r$ is the range and $\dot{r}$ is the target velocity. $\mathbf{x}_{k}$ is the target state vector at time $k . \mathbf{F}, \mathbf{G}$, and $\mathbf{H}$ are given matrices for onedimensional target tracking. $\mathbf{w}_{k}$ and $\mathbf{n}_{k}$ are the zero-mean white Gaussian noise vectors with covariance matrices $\mathbf{Q}_{k}$ and $\mathbf{N}\left(\boldsymbol{\theta}_{k}\right)$, respectively. The vector $\boldsymbol{\theta}_{k}$ characterizes the waveform parameters at time $k$.

According to Lemma 3.1 in [13], build the relationship between the receiver estimation parameter vector $\boldsymbol{\alpha}$ and measurement vector $\mathbf{y}$ through a linear transformation $\mathbf{T}$, that is, $\mathbf{y}=\mathbf{T} \boldsymbol{\alpha}$. And the measurement noise covariance matrix is dependent on waveform parameter $\boldsymbol{\theta}$ as follows:

$$
\mathbf{N}(\boldsymbol{\theta})=\frac{1}{\eta} \mathbf{T} \mathbf{J}^{-1}(\boldsymbol{\theta}) \mathbf{T},
$$

where $\mathbf{T}=\operatorname{diag}\left(c / 2, c / 2 \omega_{c}\right), \mathbf{J}$ is the Fisher information and $\operatorname{Cov}(\boldsymbol{\alpha})=\mathbf{J}^{-1}(\boldsymbol{\theta})$.

After finding the relationship between the measurement noise covariance matrix and the waveform parameter, the Kalman filter equations are dependent on $\boldsymbol{\theta}$ as follows:

$$
\begin{gathered}
\mathbf{S}_{k}\left(\boldsymbol{\theta}_{k}\right)=\mathbf{H} \mathbf{P}_{k / k-1} \mathbf{H}^{T}+\mathbf{N}\left(\boldsymbol{\theta}_{k}\right), \\
\mathbf{K}_{k}\left(\boldsymbol{\theta}_{k}\right)=\mathbf{P}_{k / k-1} \mathbf{H}^{T} \mathbf{S}_{k}^{-1}\left(\boldsymbol{\theta}_{k}\right), \\
\widehat{\mathbf{x}}_{k / k}\left(\boldsymbol{\theta}_{k}\right)=\widehat{\mathbf{x}}_{k / k-1}-\mathbf{K}_{k}\left(\boldsymbol{\theta}_{k}\right)\left(\mathbf{y}_{k}-\mathbf{H} \widehat{\mathbf{x}}_{k / k-1}\right), \\
\mathbf{P}_{k / k}\left(\boldsymbol{\theta}_{k}\right)=\mathbf{P}_{k / k-1}\left(\boldsymbol{\theta}_{k}\right)-\mathbf{K}_{k}\left(\boldsymbol{\theta}_{k}\right) \mathbf{S}_{k}\left(\boldsymbol{\theta}_{k}\right) \mathbf{K}_{k}^{T}\left(\boldsymbol{\theta}_{k}\right), \\
\widehat{\mathbf{x}}_{k+1 / k}\left(\boldsymbol{\theta}_{k}\right)=\mathbf{F} \widehat{\mathbf{x}}_{k / k}\left(\boldsymbol{\theta}_{k}\right), \\
\mathbf{P}_{k+1 / k}\left(\boldsymbol{\theta}_{k}\right)=\mathbf{F P}_{k / k}\left(\boldsymbol{\theta}_{k}\right) \mathbf{F}^{T}+\mathbf{G} \mathbf{Q}_{k} \mathbf{G}^{T} .
\end{gathered}
$$


In order to improve the tracking performance, minimizing the trace of the mean square tracking error as cost function is used to select the next transmitted waveform. That is,

$$
\boldsymbol{\theta}_{k+1}^{*}=\arg \min _{\boldsymbol{\theta}_{k+1} \in \Theta} \operatorname{Tr}\left\{\mathbf{P}_{k+1 / k+1}\left(\boldsymbol{\theta}_{k+1}\right)\right\} .
$$

In addition, minimization of the validation gate volume as another cost function is to select next transmitted waveform, which will reduce the number of false alarms in high SNR or clutter environment. So the next transmitted waveform is determined by

$$
\boldsymbol{\theta}_{k+1}^{*}=\arg \min _{\boldsymbol{\theta}_{k+1} \in \Theta} \operatorname{det}\left\{\mathbf{S}_{k+1}\left(\boldsymbol{\theta}_{k+1}\right)\right\} .
$$

When the target is intelligent enough to maximize the estimation error, it could deliberately degrade the tracking performance and even break the tracking task down. In this case, the target state model has a fictitious adversary disturbance that includes some unknown noise, which could be "smart" enough to maximize the estimation state error and decrease the target tracking performance [18]. Thus the Kalman filter and its relative optimal waveform method mentioned before are not suitable for this case. Thus, we should consider the robust tracking problem, a minimax filter based on zero-sum game is needed for target tracking, and a new method for optimal waveform will be presented in Section 3.

\section{Minimax Filter and Waveform Selection}

3.1. Minimax Filter. In order to guarantee the target tracking performance for "smart" target, the minimax filter is needed. Like $[18,19]$, the discrete linear time-invariant system in adversary disturbance which existed is expressed by

$$
\begin{gathered}
\mathbf{x}_{k+1}=\mathbf{F} \mathbf{x}_{k}+\mathbf{G w}_{k}+\mathbf{d}_{k}, \\
\mathbf{y}_{k}=\mathbf{H} \mathbf{x}_{k}+\mathbf{n}_{k},
\end{gathered}
$$

where

$$
\mathbf{d}_{k}=\mathbf{L}_{k}\left(\mathbf{H}\left(\mathbf{x}_{k}-\widehat{\mathbf{x}}_{k / k-1}\right)+\mathbf{v}_{k}\right) .
$$

Equation (12) is adversary disturbance signal which could increase the estimated error. $\mathbf{L}$ is a gain to be determined; $\mathbf{v}_{k}$ is Gaussian noise vector with zero mean and covariance matrix R. The other parameters, $\mathbf{x}, \mathbf{y}, \mathbf{H}, \mathbf{G}, \mathbf{w}$, and $\mathbf{n}$, in (10) and (11), are the same as in (4) and (5).

Based on zero-sum game, the predicted state is

$$
\widehat{\mathbf{x}}_{k+1 / k}=\mathbf{F} \widehat{\mathbf{x}}_{k / k-1}+\mathbf{K}_{k}\left(\mathbf{y}_{k}-\mathbf{H} \widehat{\mathbf{x}}_{k / k-1}\right),
$$

where $\widehat{\mathbf{x}}_{k+1 / k}$ is the predicted state, $\mathbf{K}$ is the minimax filter gain, and the prediction state error is defined by

$$
\mathbf{e}_{k / k-1}=\mathbf{x}_{k}-\widehat{\mathbf{x}}_{k / k-1} \text {. }
$$

Substituting (10) and (13) into (14), we have

$$
\mathbf{e}_{k+1 / k}=\mathbf{F}\left(\mathbf{x}_{k}-\widehat{\mathbf{x}}_{k / k-1}\right)-\mathbf{K}_{k}\left(\mathbf{y}_{k}-\mathbf{H} \widehat{\mathbf{x}}_{k / k-1}\right)+\mathbf{G w}_{k}+\mathbf{d}_{k} .
$$

Substituting (11) and (12) into (15), the final prediction error at time $k+1$ is

$$
\mathbf{e}_{k+1 / k}=(\mathbf{F}-\mathbf{K H}+\mathbf{L H}) \mathbf{e}_{k / k-1}+\mathbf{G w}_{k}+\mathbf{L} \mathbf{v}_{k}-\mathbf{K} \mathbf{n}_{k} .
$$

From (15), it can been seen that the adversary part, $\mathbf{d}_{k}$, can increase the estimation error. To prevent this, the estimation error in (16) can be decomposed as follows:

$$
\mathbf{e}_{k+1 / k}=\mathbf{e}_{k+1 / k}^{K}+\mathbf{e}_{k+1 / k}^{L},
$$

where

$$
\begin{gathered}
\mathbf{e}_{k+1 / k}^{K}=\left(\mathbf{F}-\mathbf{K}_{k} \mathbf{H}+\mathbf{L}_{k} \mathbf{H}\right) \mathbf{e}_{k / k-1}^{K}+\mathbf{G} \mathbf{w}_{k}-\mathbf{K}_{k} \mathbf{n}_{k}, \\
\mathbf{e}_{0}^{K}=\mathbf{x}_{0}, \\
\mathbf{e}_{k+1 / k}^{L}=\left(\mathbf{F}-\mathbf{K}_{k} \mathbf{H}+\mathbf{L}_{k} \mathbf{H}\right) \mathbf{e}_{k / k-1}^{L}+\mathbf{L}_{k} \mathbf{v}_{k}, \\
\mathbf{e}_{0}^{L}=\mathbf{0} .
\end{gathered}
$$

Motivated by $[18,19]$ the cost function is defined by

$$
J(\mathbf{K}, \mathbf{L})=\operatorname{trace}\left(\sum_{k=0}^{T} E\left[\left\|\mathbf{e}_{k+1 / k}^{K}\right\|^{2}-\left\|\mathbf{e}_{k+1 / k}^{L}\right\|^{2}\right]\right) .
$$

The minimax filter designed based on zero-sum game is to find the optimized filter gain $\mathbf{K}$ and the robust filter gain $\mathbf{L}$. The gain $\mathbf{K}$ should be optimized to minimize the $J$ so that the tracking performance is better, since the prediction error $\mathbf{e}_{k+1 / k}^{K}$ is relative to noises of $\mathbf{w}_{k}$ and $\mathbf{n}_{k}$. The gain $\mathbf{L}$ should be optimized to maximize the $J$, since the $\mathbf{e}_{k+1 / k}^{L}$ is relative to the noise of $\mathbf{v}_{k}$, which makes the worst possible disturbance. Let $\mathbf{K}^{*}$ and $\mathbf{L}^{*}$ denote the optimized gains, which satisfies a saddle-point equilibrium, that is,

$$
J\left(\mathbf{K}^{*}, \mathbf{L}\right) \leq J\left(\mathbf{K}^{*}, \mathbf{L}^{*}\right) \leq J\left(\mathbf{K}, \mathbf{L}^{*}\right) .
$$

To solve (20), the cost function (19) needs to be written in a more convenient form. Define $\mathbf{Z}_{k}$ as follows:

$$
\mathbf{Z}_{k}=\mathbf{F}-\mathbf{K}_{k} \mathbf{H}+\mathbf{L}_{k} \mathbf{H} \text {. }
$$

Let $\mathbf{P}_{k+1 / k}^{K}=E\left[\left(\mathbf{e}_{k+1 / k}^{K}\right)\left(\mathbf{e}_{k+1 / k}^{K}\right)^{T}\right]$ and $\mathbf{P}_{k+1 / k}^{L}=$ $E\left[\left(\mathbf{e}_{k+1 / k}^{L}\right)\left(\mathbf{e}_{k+1 / k}^{L}\right)^{T}\right]$; we have

$$
\begin{gathered}
\mathbf{P}_{k+1 / k}^{K}=\mathbf{Z}_{k} \mathbf{P}_{k / k-1}^{K} \mathbf{Z}_{k}^{T}+\mathbf{G Q}_{k} \mathbf{G}^{T}+\mathbf{K}_{k} \mathbf{N} \mathbf{K}_{k}^{T}, \\
\mathbf{P}_{k+1 / k}^{L}=\mathbf{Z}_{k} \mathbf{P}_{k / k-1}^{L} \mathbf{Z}_{k}^{T}+\mathbf{L}_{k} \mathbf{R} \mathbf{L}_{k}^{T} .
\end{gathered}
$$

The cost function (19) can be rewritten by

$$
J(\mathbf{K}, \mathbf{L})=\operatorname{trace}\left(\sum_{k=0}^{T} \mathbf{P}_{k+1 / k}\right),
$$

where $\mathbf{P}_{k+1 / k}=\mathbf{P}_{k+1 / k}^{K}-\mathbf{P}_{k+1 / k}^{L}=\mathbf{Z}_{k} \mathbf{P}_{k / k-1} \mathbf{Z}_{k}^{\%}+\mathbf{G Q}_{k} \mathbf{G}^{T}+$ $\mathbf{K}_{k} \mathbf{N} \mathbf{K}_{k}^{T}-\mathbf{L}_{k} \mathbf{R} \mathbf{L}_{k}^{T}$.

Let

$$
\mathbf{U}_{k}=\mathbf{G Q}_{k} \mathbf{G}^{T}+\mathbf{K}_{k} \mathbf{N} \mathbf{K}_{k}^{T}-\mathbf{L}_{k} \mathbf{R} \mathbf{L}_{k}^{T} .
$$


Then $\mathbf{P}_{k+1 / k}=\mathbf{Z}_{k} \mathbf{P}_{k / k-1} \mathbf{Z}_{k}^{T}+\mathbf{U}_{k}$. According to Theorem 1 in [18], the game equilibrium is derived by

$$
\begin{gathered}
\mathbf{K}_{k}^{*}=\mathbf{F} \boldsymbol{\Sigma}_{k} \mathbf{H}^{T} \mathbf{N}^{-1}, \\
\mathbf{L}_{k}^{*}=\mathbf{F} \boldsymbol{\Sigma}_{k} \mathbf{H}^{T} \mathbf{R}^{-1}, \\
\mathbf{\Sigma}_{k}^{-1}=\mathbf{P}_{k / k-1}^{-1}+\mathbf{H}^{T}\left(\mathbf{N}^{-1}-\mathbf{R}^{-1}\right) \mathbf{H} .
\end{gathered}
$$

After obtaining the game equilibrium $\left(\mathbf{K}_{k}^{*}, \mathbf{L}_{k}^{*}\right)$, substitute $\mathbf{K}_{k}^{*}$ and $\mathbf{L}_{k}^{*}$ into (21) and (24), respectively. the covariance matrix $\mathbf{P}_{k+1 / k}$ can be expressed by

$$
\mathbf{P}_{k+1 / k}=\mathbf{F} \Sigma_{k} \mathbf{F}^{T}+\mathbf{G Q G}^{T} .
$$

Finally, the minimax filter based on zero-sum game equations is

$$
\begin{gathered}
\widehat{\mathbf{x}}_{k+1 / k}=\mathbf{F} \widehat{\mathbf{x}}_{k / k-1}+\mathbf{K}_{k}^{*}\left(\mathbf{y}_{k}-\mathbf{H} \widehat{\mathbf{x}}_{k / k-1}\right) \\
\mathbf{P}_{k+1 / k}=\mathbf{F} \boldsymbol{\Sigma}_{k} \mathbf{F}^{T}+\mathbf{G Q G} \mathbf{G}^{T} \\
\boldsymbol{\Sigma}_{k}^{-1}=\mathbf{P}_{k / k-1}^{-1}+\mathbf{H}^{T}\left(\mathbf{N}^{-1}-\mathbf{R}^{-1}\right) \mathbf{H} \\
\mathbf{K}_{k}^{*}=\mathbf{F} \boldsymbol{\Sigma}_{k} \mathbf{H}^{T} \mathbf{N}^{-1} \\
\mathbf{L}_{k}^{*}=\mathbf{F} \boldsymbol{\Sigma}_{k} \mathbf{H}^{T} \mathbf{R}^{-1}
\end{gathered}
$$

The minimax filter based on the game theory is suitable for the robust target tracking. However, the minimax filter only considers the tracking performance in the receiver. in order to improve the robust tracking performance better, the transmitter waveform could be considered for "smart" target, and then, we will deliberate the waveform selection for robust target tracking

3.2. Waveform Selection. According to the review of the control approach in [13], the minimax filter is related to the waveform parameter by the measurement noise covariance. So considering the waveform parameter, (27) could be rewritten as

$$
\begin{gathered}
\widehat{\mathbf{x}}_{k+1 / k}\left(\boldsymbol{\theta}_{k}\right)=\mathbf{F} \widehat{\mathbf{x}}_{k / k-1}+\mathbf{K}_{k}^{*}\left(\boldsymbol{\theta}_{k}\right)\left(\mathbf{y}_{k}-\mathbf{H} \widehat{\mathbf{x}}_{k / k-1}\right), \\
\mathbf{P}_{k+1 / k}\left(\boldsymbol{\theta}_{k}\right)=\mathbf{F} \boldsymbol{\Sigma}_{k}\left(\boldsymbol{\theta}_{k}\right) \mathbf{F}^{T}+\mathbf{G Q G} \mathbf{G}^{T}, \\
\boldsymbol{\Sigma}_{k}^{-1}\left(\boldsymbol{\theta}_{k}\right)=\mathbf{P}_{k / k-1}^{-1}+\mathbf{H}^{T}\left(\mathbf{N}^{-1}\left(\boldsymbol{\theta}_{k}\right)-\mathbf{R}^{-1}\right) \mathbf{H}, \\
\mathbf{K}_{k}^{*}\left(\boldsymbol{\theta}_{k}\right)=\mathbf{F} \boldsymbol{\Sigma}_{k}\left(\boldsymbol{\theta}_{k}\right) \mathbf{H}^{T} \mathbf{N}^{-1}\left(\boldsymbol{\theta}_{k}\right), \\
\mathbf{L}_{k}^{*}\left(\boldsymbol{\theta}_{k}\right)=\mathbf{F} \boldsymbol{\Sigma}_{k}\left(\boldsymbol{\theta}_{k}\right) \mathbf{H}^{T} \mathbf{R}^{-1}
\end{gathered}
$$

where $\boldsymbol{\theta}_{k}$ is the waveform parameter vector. The minimax filter in (28) contains the transmitted waveform parameters, which is similar to the results of (27) when the transmitted waveform parameter $\boldsymbol{\theta}_{k}$ is fixed. However, it does not indicate what is the relationship between the next step transmitted waveform and corresponding tracking performance. Equation (27) just found that the current waveform parameter impects the next step prediction error.
Our aim is to find the relationship between the transmitted waveforms and its corresponding tracking performance, build the optimization criterion, and select the optimal waveform to improve the robust tracking performance better compared to the minimax filter.

Like standard Kalman filter

$$
\begin{gathered}
\widehat{\mathbf{x}}_{k / k}=\widehat{\mathbf{x}}_{k / k-1}+\mathbf{G}_{k / k-1}\left(\mathbf{y}_{k}-\mathbf{H} \widehat{\mathbf{x}}_{k / k-1}\right) \\
\widehat{\mathbf{x}}_{k+1 / k}=\mathbf{F} \widehat{\mathbf{x}}_{k / k}
\end{gathered}
$$

where $\widehat{\mathbf{x}}_{k / k}$ is the estimated state and $\mathbf{G}_{k / k-1}$ is the filter gain. Substituting (29) into (30), we have

$$
\widehat{\mathbf{x}}_{k+1 / k}=\mathbf{F} \widehat{\mathbf{x}}_{k / k-1}+\mathbf{F G}_{k / k-1}\left(\mathbf{y} \mathbf{H} \widehat{\mathbf{x}}_{k / k-1}\right) .
$$

Compared to (13), the relationship between the two gains, $\mathbf{G}$ and $\mathbf{K}$, is

$$
\mathbf{G}_{k / k-1}=\mathbf{F}^{-1} \mathbf{K}_{k} \text {. }
$$

The estimated state error is defined by

$$
\mathbf{e}_{k+1 / k+1}=\widehat{\mathbf{x}}_{k+1}-\widehat{\mathbf{x}}_{k+1 / k+1} \text {. }
$$

According to (11), (29) and (33), the estimated state error could be derived by

$$
\mathbf{e}_{k+1 / k+1}=\left(\mathbf{I}-\mathbf{G}_{k+1 / k} \mathbf{H}\right) \mathbf{e}_{k+1 / k}-\mathbf{G}_{k+1 / k} \mathbf{n}_{k+1},
$$

where $\mathbf{I}$ is the identity matrix.

The covariance of the estimated state error is defined by

$$
\mathbf{P}_{k+1 / k+1}=E\left(\mathbf{e}_{k+1 / k+1} \mathbf{e}_{k+1 / k+1}^{T}\right) \text {. }
$$

Substituting (34) into (35) and considering the transmitted waveform parameter, we have

$$
\begin{aligned}
\mathbf{P}_{k+1 / k+1}\left(\boldsymbol{\theta}_{k+1}\right)= & \mathbf{M}_{k+1 / k} \mathbf{P}_{k+1 / k}^{K} \mathbf{M}_{k+1 / k}^{T} \\
& +\mathbf{M}_{k+1 / k} \mathbf{P}_{k+1 / k}^{L} \mathbf{M}_{k+1 / k}^{T} \\
& +\mathbf{G}_{k+1 / k} \mathbf{N}\left(\boldsymbol{\theta}_{k+1}\right) \mathbf{G}_{k+1 / k}^{T},
\end{aligned}
$$

where $\mathbf{M}_{k+1 / k}=\left(\mathbf{I}-\mathbf{G}_{k+1 / k} \mathbf{H}\right)$.

Now, the relationship between the next transmitted waveform parameter and its corresponding target tracking performance is built. Thus, the optimization criterion is to select one transmitted waveform parameter from the parameter database to minimize the trace of the estimated state covariance, that is

$$
\boldsymbol{\theta}_{k+1}^{*}=\min _{\boldsymbol{\theta}_{k+1} \in \Theta} \operatorname{tr}\left\{\mathbf{P}_{k+1 / k+1}\left(\boldsymbol{\theta}_{k+1}\right)\right\} .
$$

When the next optimal transmitted waveform parameter is selected, the measurement noise covariance matrix is known, which improves the robust tracking performance.

The proposed optimal waveform selection can be summarized as follows.

(1) When the minimax filter gets the target state information at time $k$ through (28), compute the gain $\mathbf{G}_{k+1 / k}$ through (32). 
(2) Compute the measurement covariance matrix $\mathbf{N}\left(\boldsymbol{\theta}_{k+1}\right)$ through (6) for every waveform parameter stored in the database.

(3) According to (36), compute the trace of every estimation error covariance for every waveform parameter and find the minimization of the values. The waveform parameter, which is corresponding to the minimization value, is the optimal selection for next transmitted waveform.

\section{Simulation Results}

The proposed method is examined in this section. The discrete linear time-invariant system matrices are followed by

$$
\begin{gathered}
\mathbf{F}=\left[\begin{array}{lll}
1 & 1 & 0.5 \\
0 & 1 & 1 \\
0 & 0 & 1
\end{array}\right], \\
\mathbf{G}=\left[\begin{array}{lll}
1 & 0 & 0 \\
0 & 1 & 0 \\
0 & 0 & 1
\end{array}\right], \\
\mathbf{H}=\left[\begin{array}{lll}
1 & 0 & 0 \\
0 & 1 & 0
\end{array}\right], \\
\mathbf{Q}=\left[\begin{array}{ccc}
0.001 & 0 & 0 \\
0 & 0.01 & 0 \\
0 & 0 & 0.01
\end{array}\right], \\
\mathbf{S}=\left[\begin{array}{cc}
0.9 & 0 \\
0 & 0.9
\end{array}\right] .
\end{gathered}
$$

The normal target trajectory is as follows:

$$
x=100+0.2 t+0.1 t^{2}+\cos (5 \pi t) .
$$

Considering the adversary disturbance, the intelligent target model is

$$
\mathbf{x}_{k+1}=\mathbf{F} \mathbf{x}_{k}+0.08\left(\mathbf{x}_{k}+\widehat{\mathbf{x}}_{k / k-1}\right)
$$

Some simulation parameters are adopted from [13], the carrier frequency $\omega_{c}$ is $25 \mathrm{kHz}$, and the speed of the transmitted signal $c$ is $1500 \mathrm{~m} / \mathrm{s}$. the return pulse signal-to-noise ratio $\eta$ is modeled by

$$
\eta=\left(\frac{1000}{r}\right)^{4} \eta_{1000}
$$

where $\eta$ is the returned pulse signaltonoise for a target at $1000 \mathrm{~m} . \eta_{1000} \approx 0 \mathrm{~dB} . r$ is the target range. The triangularshaped pulse belongs to amplitude-only modulation that is used as transmitted pulse. The waveform parameter is the wavelength $\lambda$. We set the parameter database as follows:

$$
\lambda=[0.1: 0.05: 0.3],
$$

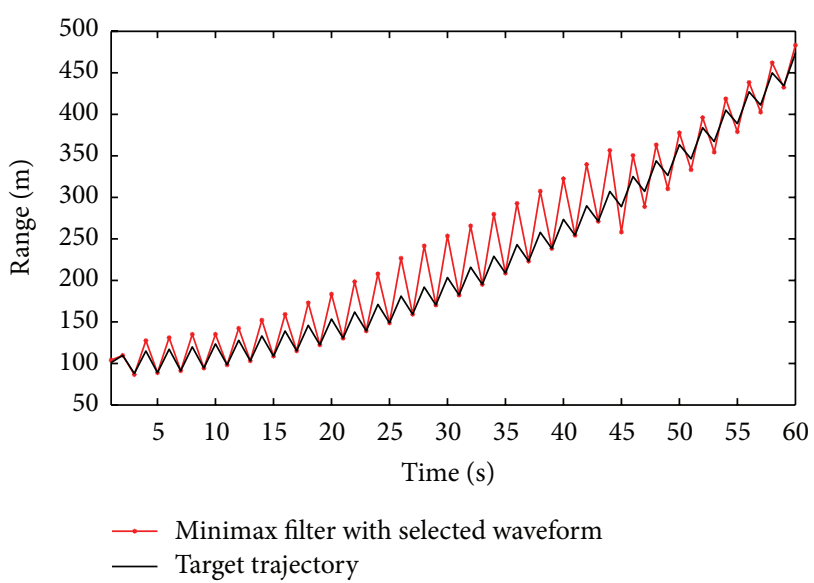

FIGURE 1: Estimation of the intelligent target by the minimax filter with selected waveform.

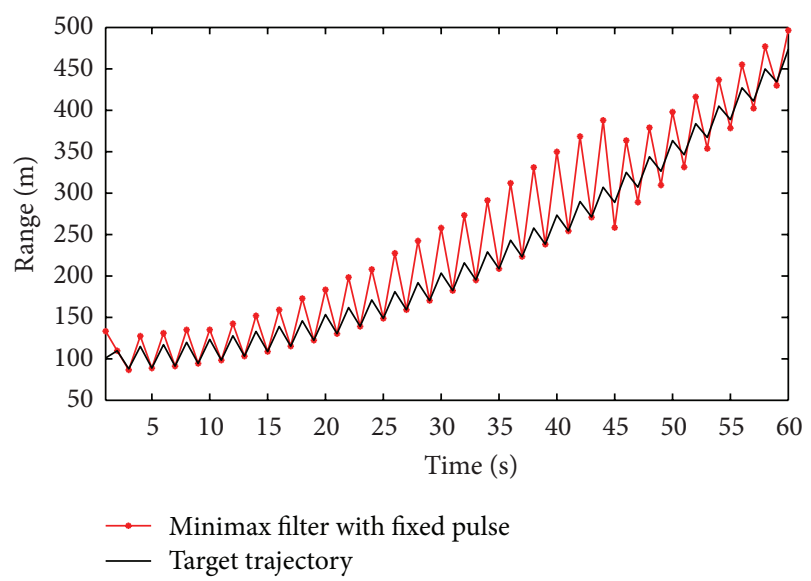

FIGURE 2: Estimation of the intelligent target by the minimax filter with fixed waveform.

where 0.05 is the step length. The relationship between the wavelength $\lambda$ and the measurement noise covariance is [13]

$$
R\left(\lambda_{k+1}\right)=\left[\begin{array}{cc}
\frac{c^{2} \lambda_{k+1}^{2}}{(12 \eta)} & 0 \\
0 & \frac{5 c^{2}}{\left(2 \omega_{c}^{2} \lambda_{k+1}^{2} \eta\right)}
\end{array}\right] .
$$

Firstly, the minimax filters with selected waveform and fixed waveform are used to estimate the intelligent target, respectively. The estimation trajectory of the minimax filter with selected waveform and the target trajectory are shown in Figure 1. The estimation trajectory of the minimax filter with fixed waveform and the target trajectory are shown in Figure 2. It can be seen that two filters can overcome the adversary noise that deliberately maximizes the estimation error and estimate the true target trajectory well.

The performance of the two minimax filters with selected waveform and fixed waveform is shown in Figures 3 and 4. Figure 3 shows the the pulse length is selected in every time in the minimax filter with selected waveform. Figure 4 shows 


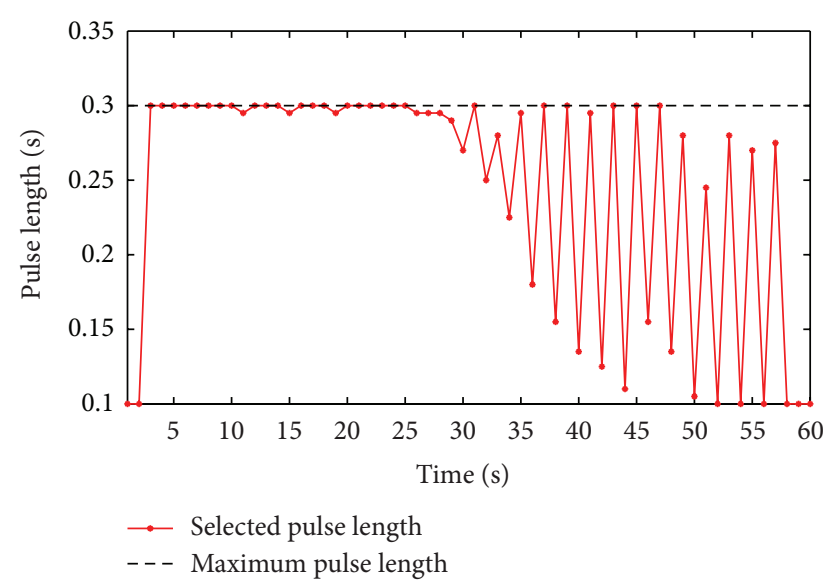

Figure 3: Parameter selection.

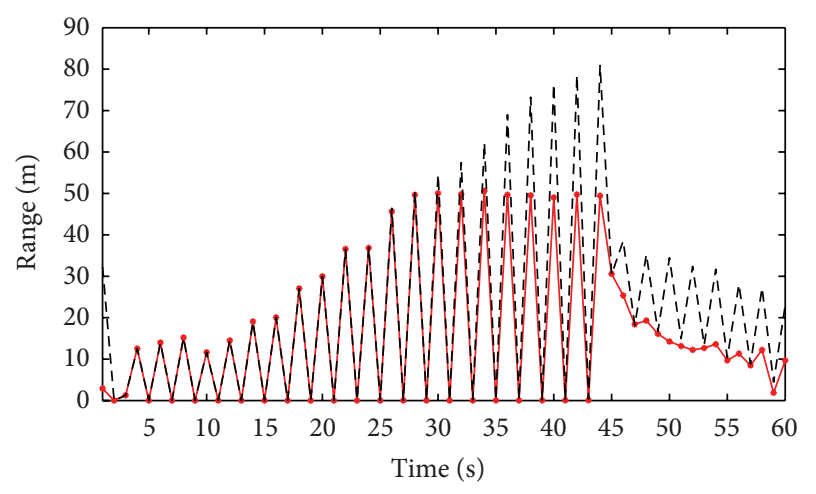

$\rightarrow$ Minimax filter with selected waveform

- - - Minimax filter with fixed waveform

FIGURE 4: Estimation errors.

the position errors in the two different minimax filters. It can be seen that sometimes, the minimax filter with selected waveform selects the parameters which are the same as the one with fixed waveform. the position errors by the two filters are almost the same. While, in other times, the position error by the minimax filter with selected waveform is smaller than the one by the minimax filter with fixed waveform, since the measurement noise covariance is impacted by the transmitted waveform parameter. When the target range is known, the measurement noise covariance is only changed by the different waveform parameters. The system will select the "best" waveform in order to minimize the the trace of the estimated state covariance. however, the minimax filter with fixed waveform generates the fixed trace of the estimated state covariance when the target range is know

\section{Conclusion}

This paper focuses on the optimal waveform selection for robust target tracking. When the target is assumed to have the "smart" ability, which could increase the estimation error and degrade the target tracking performance, the minimax filter based on the game theory could address the robust tracking problem well from the receiver. On this basis, we improve the minimax filter combining with the waveform selection from the transmitter and derive its the estimation error covariance. Then, according to the relationship between the waveform parameter and measurement noise covariance, the estimation error covariance is related to waveform parameter. Build the optimization criterion that minimizes the estimation error covariance by selecting the waveform parameter at every transmission. The simulation results show the proposed method make the performance of the robust target tracking better than the minimax filter with fixed waveform based game theory.

\section{Acknowledgments}

This work was supported by the National Natural Science Foundation of China (no. 61004052 and no. 61104005), the Natural Science Foundation of Hebei Province (no. F2013501075), the Fundamental Research Funds for the Central Universities (no. N110323005) and the Doctoral Scientific Research Foundation of Liaoning Province (no. 20131030).

\section{References}

[1] S. Haykin, "Cognitive radar: a way of the future," IEEE Signal Processing Magazine, vol. 23, no. 1, pp. 30-40, 2006.

[2] S. Haykin, "Cognition is the key to the next generation of radar systems," in Proceedings of the 13th IEEE Digital Signal Processing Workshop and 5th IEEE Signal Processing Education Workshop (DSP/SPE '09), pp. 463-467, January 2009.

[3] S. U. Pillai, D. C. Youla, H. S. Oh, and J. R. Guerci, "Optimum transmit-receiver design in the presence of signal-dependent interference and channel noise," in Proceedings of the 33rd Asilomar Conference on Signals, Systems, and Computers, vol. 2, pp. 870-875, Pacific Grove, Calif, USA, 1999.

[4] S. U. Pillai, H. S. Oh, D. C. Youla, and J. R. Guerci, "Optimum transmit-receiver design in the presence of signal-dependent interference and channel noise," IEEE Transactions on Information Theory, vol. 46, no. 2, pp. 577-584, 2000.

[5] J. R. Guerci and S. U. Pillai, "Theory and application of optimum transmit-receive radar," in Proceedings of the IEEE International Radar Conference, pp. 705-710, Alexandria, Va, USA, May 2000.

[6] J. R. Guerci and S. U. Pillai, "Adaptive transmission radar: the next "wave"?" in Proceedings of the IEEE National Aerospace and Electronics Conference (NAECON '00), pp. 779-786, Dayton, Ohio, USA, October 2000.

[7] M. R. Bell, "Information theory and radar waveform design," IEEE Transactions on Information Theory, vol. 39, no. 5, pp. 1578-1597, 1993.

[8] S. M. Sowelam and A. H. Tewfik, "Waveform selection in radar target classification," IEEE Transactions on Information Theory, vol. 46, no. 3, pp. 1014-1029, 2000.

[9] S. Kay, "Waveform design for multistatic radar dsetection," IEEE Transactions on Aerospace and Electronic Systems, vol. 45, no. 3, pp. 1153-1166, 2009.

[10] N. A. Goodman, P. R. Venkata, and M. A. Neifeld, "Adaptive waveform design and sequential hypothesis testing for target recognition with active sensors," IEEE Journal on Selected Topics in Signal Processing, vol. 1, no. 1, pp. 105-113, 2007. 
[11] R. A. Romero, J. Bae, and N. A. Goodman, "Theory and application of SNR and mutual information matched illumination waveforms," IEEE Transactions on Aerospace and Electronic Systems, vol. 47, no. 2, pp. 912-927, 2011.

[12] Y. Wei, H. Meng, Y. Liu, and X. Wang, "Radar phase-coded waveform design for extended target recognition under detection constraints," in Proceedings of the IEEE Radar Conference, pp. 1074-1079, May 2011.

[13] D. J. Kershaw and R. J. Evans, "Optimal waveform selection for tracking systems," IEEE Transactions on Information Theory, vol. 40, no. 5, pp. 1536-1550, 1994.

[14] S. D. Howard, S. Suvorova, and A. Nehorai, "Waveform libraries for radar tracking applications," in Proceedings of the International Conference on Waveform Diversity and Design, pp. 1-5, Edinburgh, UK, November 2004.

[15] S. Suvorova, S. D. Howard, W. Moran, and R. J. Evans, "Waveform libraries for radar tracking applications: maneuvering targets," in Proceedings of the 40th Annual Conference on Information Sciences and Systems (CISS '06), pp. 1424-1428, Princeton, NJ, USA, March 2006.

[16] D. J. Kershaw and R. J. Evans, "Waveform selective probalilistic data association," IEEE Transactions on Aerospace and Electronic Systems, vol. 33, pp. 1180-1188, 1997.

[17] S. P. Sira, A. Papandreou-Suppappola, and D. Morrell, "Dynamic configuration of time-varying waveforms for agile sensing and tracking in clutter," IEEE Transactions on Signal Processing, vol. 55, no. 7, pp. 3207-3217, 2007.

[18] D. Gu, "A game theory approach to target tracking in sensor networks," IEEE Transactions on Systems, Man, and Cybernetics, Part B, vol. 41, no. 1, pp. 2-13, 2011.

[19] D. Simon, "A game theory approach to constrained minimax state estimation," IEEE Transactions on Signal Processing, vol. 54, no. 2, pp. 405-412, 2006. 


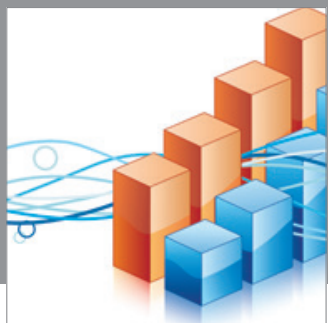

Advances in

Operations Research

mansans

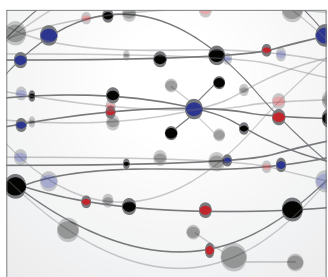

The Scientific World Journal
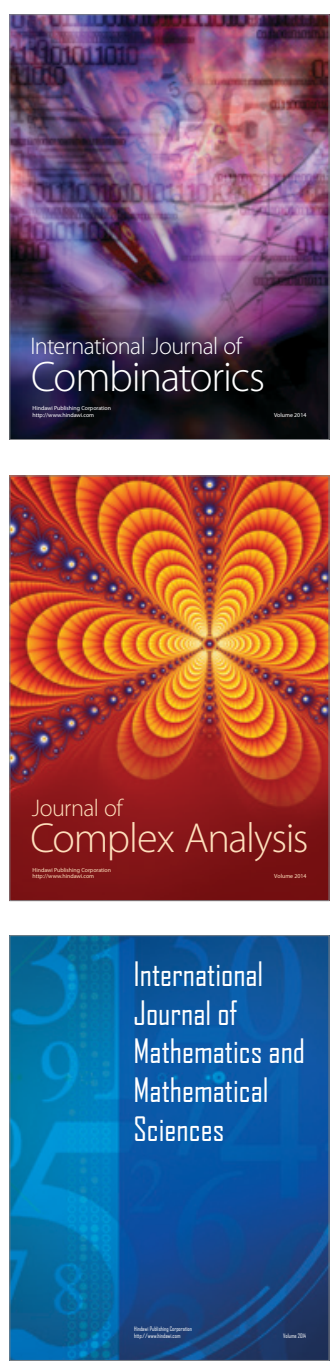
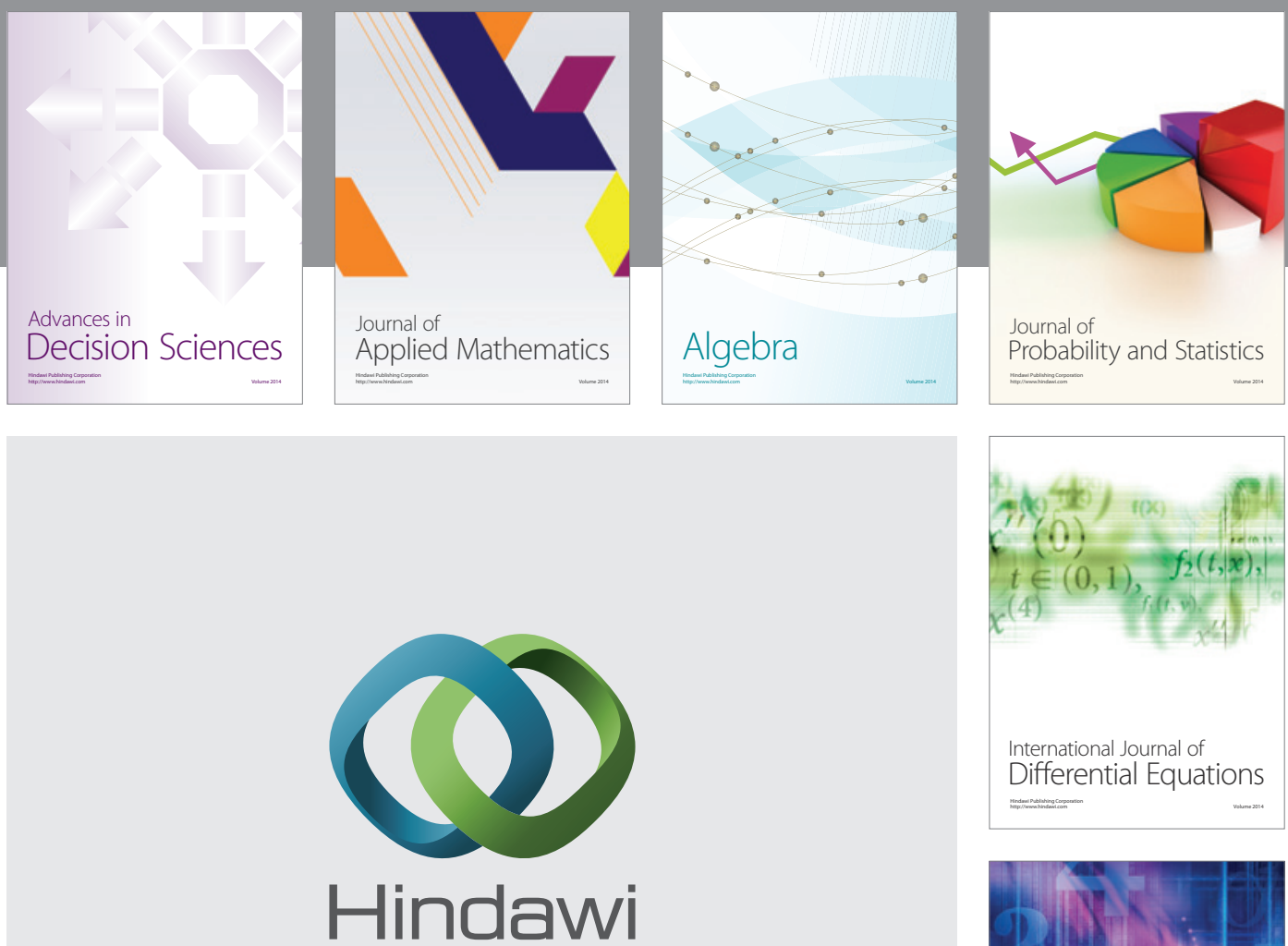

Submit your manuscripts at http://www.hindawi.com
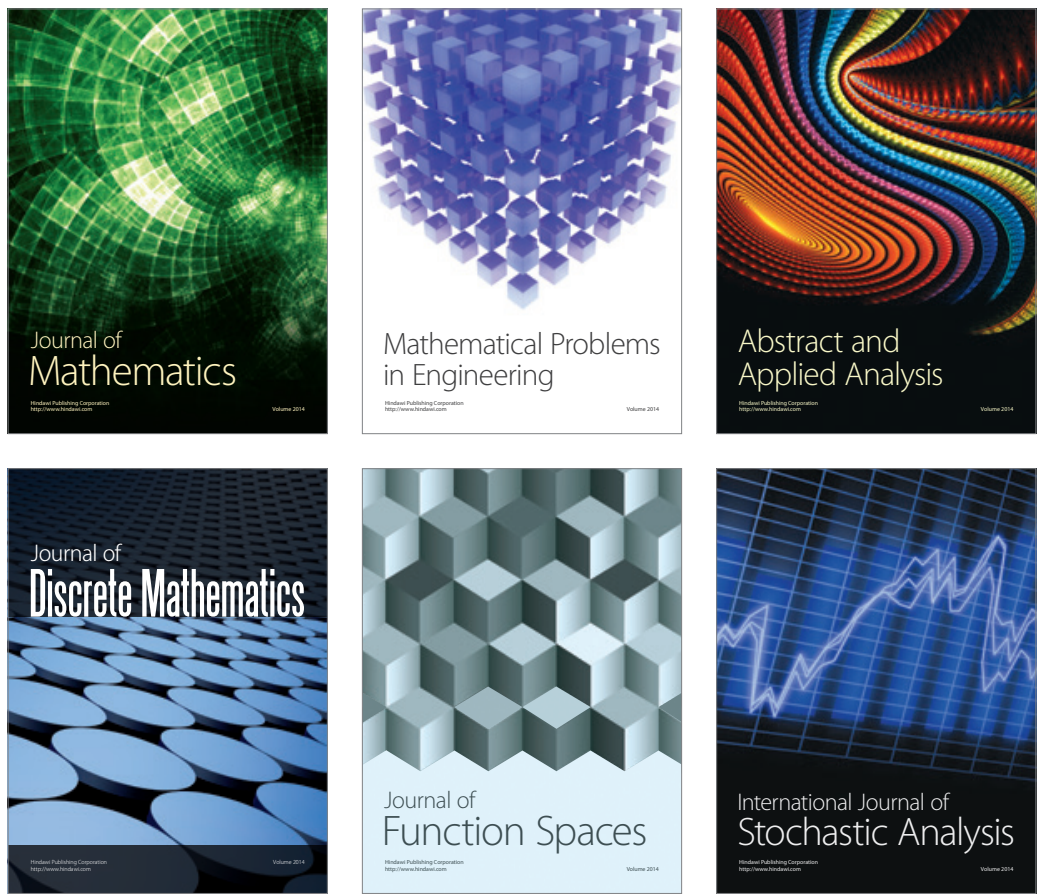

Journal of

Function Spaces

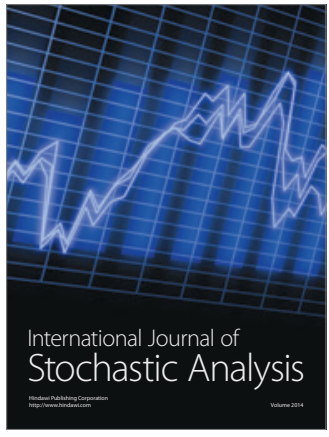

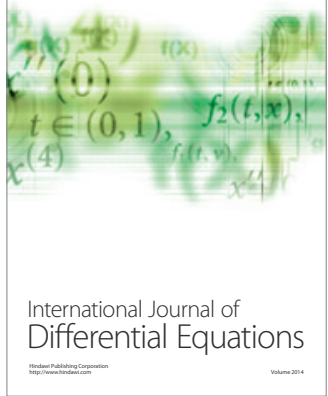
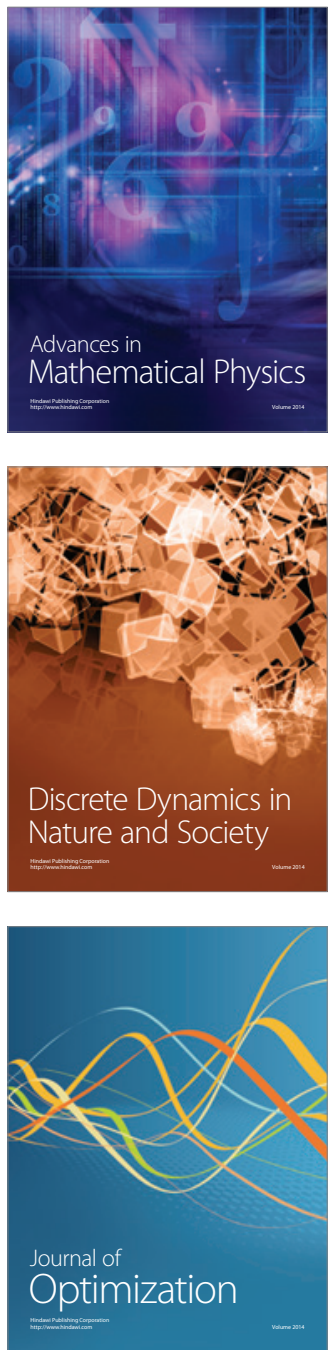